\title{
Endoscopic Ultrasound-Guided Fiducial Placement for Stereotactic Body Radiation Therapy in Pancreatic Malignancy
}

\author{
Seong-Hun Kim ${ }^{1}$ and Eun Ji Shin ${ }^{2}$ \\ ${ }^{1}$ Department of Internal Medicine, Research Institute of Clinical Medicine of Jeonbuk National University-Biomedical Research Institute \\ of Jeonbuk National University Hospital, Jeonju, Korea, ${ }^{2}$ Division of Gastroenterology and Hepatology, Johns Hopkins University, \\ Baltimore, MD, USA
}

Stereotactic body radiation therapy (SBRT) is an important treatment option for pancreatic cancer, which is known to be one of the malignancies with the worst prognosis. However, the high radiation doses delivered during SBRT may cause damage to adjacent radiosensitive organs. To minimize such damage, fiducial markers are used for localization during SBRT for pancreatic cancer. The development of endoscopic ultrasound (EUS) has enabled fiducial markers to be inserted into the pancreas using an EUS fine-needle aspiration (FNA) needle, unlike in the past when percutaneous placement was generally performed. For successful EUS-guided fiducial marker placement, it is necessary for the fiducial markers to be loaded within the EUS-FNA needles to have a low probability of complications and a low migration risk, and to be stably observed in SBRT imaging. A systematic review has shown that the technical success rate of EUS-guided fiducial marker placement is $96.27 \%$, whereas the fiducial marker migration and adverse event rates are $4.33 \%$ and $4.85 \%$, respectively. Nonetheless, standardized techniques for fiducial marker placement and the characteristics of optimal fiducial markers have not yet been established. This review will introduce the characteristics (e.g., materials and shapes) of fiducial markers used in fiducial marker placement for pancreatic cancer and will discuss conventional techniques along with their success rates, difficulties, and adverse events. Clin Endosc 2021;54:314-323

Key Words: Endoscopic ultrasonography; Fiducial marker; Pancreatic cancer; Stereotactic body radiotherapy; Technique

\section{INTRODUCTION}

The American Cancer Society has reported that pancreatic cancer is the fourth leading cause of cancer death in both men and women and is one of the malignancies with the worst prognosis. ${ }^{1}$ Among patients diagnosed with pancreatic cancer, only $10 \%$ are eligible to undergo surgery, whereas $30-40 \%$ are categorized into the borderline resectable or locally advanced group and the rest into the metastatic disease group. ${ }^{2}$ As a ther-

Received: March 16, 2021 Revised: May 5, 2021

Accepted: May 7, 2021

Correspondence: Eun Ji Shin

Division of Gastroenterology and Hepatology, Johns Hopkins School of Medicine,

Sheikh Zayed Tower, Suite 7125H, Baltimore, MD 21287, USA

Tel: +1-410-614-0950, Fax: +1-443-683-8333, E-mail: eshin3@jhmi.edu

ORCID: https://orcid.org/0000-0003-0624-8149

It is the invited review article.

cc This is an Open Access article distributed under the terms of the Creative Commons Attribution Non-Commercial License (http://creativecommons.org/ licenses/by-nc/3.0) which permits unrestricted non-commercial use, distribution, and reproduction in any medium, provided the original work is properly cited. apeutic option for pancreatic cancer, radiation therapy may decrease the recurrence risk; however, it tends to be accompanied by adverse effects and to have a prolonged treatment duration with irradiation with standard fractions. ${ }^{3-7}$ With stereotactic body radiation therapy (SBRT), the treatment duration is reduced, as $5 \mathrm{~Gy}$ of radiation per fraction is delivered to the target organ for a shorter period (i.e., between one and six sessions) compared with the standard radiation therapy, while still effectively improving the local control of pancreatic cancer. ${ }^{8}$ However, the high dose of radiation used in SBRT needs to be seriously considered, as it can cause damage to normal tissues adjacent to the target site (i.e., organs at risk [OAR]). ${ }^{8}$ Accordingly, it is essential to accurately target the lesion to reduce the radiation dose to OAR; however, it is particularly challenging to locate the tumor site in the pancreas on conebeam computed tomography (CT) imaging generally used in radiation therapy, unlike on planning CT. For this reason, it has been reported that SBRT is associated with a rate of toxicity to normal gastrointestinal organs of $>10 \% .{ }^{8}$ In an effort to 
reduce OAR toxicity, fiducial markers were developed to aid in tumor localization. Fiducial markers providing high contrast are easily observed even on cone-beam CT with no contrast enhancement, which would help accurately target the tumor during SBRT, thereby leading to a decreased risk of damage to adjacent organs. ${ }^{9}$ A recent study compared the outcomes between conventional SBRT and SBRT with fiducial marker placement in unresectable pancreatic cancer. No significant differences were observed in local recurrence, overall survival, surgical margins, and lymphovascular invasion between the two methods. In terms of toxicity, patients who underwent SBRT with fiducial marker placement had less abdominal pain but more fatigue and nausea. However, other major toxicities, such as vomiting, diarrhea, and constipation, were similar between the two groups. Notably, this study was a retrospective cohort study at a single institution and the SBRT protocol and fiducial marker insertion methods were different between the two groups. In addition, the statistical power was insufficient owing to the small number of cases. Therefore, well-designed randomized prospective studies are needed in the future. ${ }^{10}$

Three different methods are available for inserting fiducial markers into the lesion: surgical insertion, percutaneous insertion, and endoscopic ultrasound (EUS)-guided insertion. The organs in which fiducial marker placement was first attempted include the prostate, vertebrae, and lungs because these organs are relatively easy to target using the percutaneous method. ${ }^{11}$ Conversely, the pancreas is relatively challenging to access percutaneously, with a higher risk of adjacent organ damage or vascular injury. In addition, the surgical method is not optimal because of its invasiveness. ${ }^{11}$ In contrast, EUS provides high-resolution images, allowing for real-time observation of the pancreas from the stomach and duodenum. During the last three decades, EUS interventions have enabled surgeons to access any desired site in the pancreas. ${ }^{12}$ Since the first case report published in 2006, EUS-guided fiducial marker placement has become the preferred method for fiducial placement in patients with pancreatic cancer. ${ }^{2,11-15}$ However, standardized techniques and information on the characteristics of optimal fiducial markers have not been established. Thus, this review will discuss the characteristics of fiducial markers used in EUS-guided fiducial placement for pancreatic cancer, the existing techniques that have already been applied, and the success rates and adverse events of each technique.

\section{Features of fiducial marker types for EUS-guided fiducial placement}

Optimal fiducial markers for placement in the human body need to be safe and easily observed with imaging modalities such as cone-beam CT during SBRT. To increase the visibility of a fiducial marker in the imaging method commonly used in SBRT (i.e., cone-beam CT), the fiducial marker should provide high contrast and produce negligible artifacts. Furthermore, it should have a structure that provides comfort and safety in terms of technique during EUS-guided fiducial placement, as well as helps reduce complications and minimize migration. ${ }^{2,9,16}$

Fiducial markers are broadly divided into solid and liquid types, and the solid type is subdivided into three different materials: gold, platinum, and carbon (Table 1). Among these, solid gold markers have the most data available from previous

Table 1. Types of FDA-Certified Fiducial Markers

\begin{tabular}{|c|c|c|c|c|c|}
\hline Company & Fiducial marker & Material & $\begin{array}{l}\text { Diameter } \\
(\mathbf{m m})\end{array}$ & $\begin{array}{l}\text { Length } \\
(\mathrm{mm})\end{array}$ & Shape \\
\hline Best Medical International (USA) & Loose Gold Marker & Gold & 0.80 & 3 & Cylindrical \\
\hline Naslund Medical AB (Sweden) & Gold Anchor & Gold & 0.28 & 10 or 20 & Folded line shape (ball shape) \\
\hline RadioMed Corporation/IBA (Germany) & Visicoil & Gold & 0.35 & 10 & Helical coil \\
\hline Cook Medical (USA) & $\begin{array}{c}\text { EchoTip Ultra Fiducial } \\
\text { Needle }\end{array}$ & Gold & 0.43 & 5 & Step shape \\
\hline Medtronic (USA) & $\begin{array}{c}\text { Beacon FNF Preloaded } \\
\text { Needle }\end{array}$ & Gold & 0.43 & 5 & $\begin{array}{c}\text { Cylindrical (knurled exterior } \\
\text { design) }\end{array}$ \\
\hline RadioMed Corporation/IBA (Germany) & Visicoil MR & Platinum & 0.35 or 0.75 & 5 or 10 & Helical coil \\
\hline Boston Scientific (USA) & LumiCoil & Platinum & 0.46 & 5 or 10 & $\begin{array}{l}\text { Coiled outer sheath, figure-of- } \\
\text { eight or straight shape }\end{array}$ \\
\hline Carbon Medical Technologies Inc. (USA) & Acculoc Carbon Marker & Carbon & 1.00 & 3 & Cylindrical \\
\hline
\end{tabular}

FDA, Food and Drug Administration 
studies (Table 2), 2,11,15,17-26

Gold fiducial markers approved by the Food and Drug Administration (FDA) include the following: 1) Gold Anchor manufactured by Naslund Medical AB (Huddinge, Sweden), 2) Visicoil from RadioMed Corporation/IBA (RadioMed Corporation; Bartlett, TN, USA/IBA; Schwarzenbruck, Germany), 3) Loose Gold Marker from Best Medical International (Springfield, VA, USA), 4) EchoTip Ultra Fiducial Needle from Cook Medical (Bloomington, IN, USA), and 5) Beacon FNF Preloaded Needle from Medtronic (Minneapolis, MN, USA) (Table 1 ). ${ }^{2}$ Gold fiducial markers are available in diameters of $0.28,0.35,0.43$, and $0.8 \mathrm{~mm}$, and a 19 -gauge needle is required for markers with a diameter of $>0.75 \mathrm{~mm}$. In addition, they have variable lengths of 3,5,10, and $20 \mathrm{~mm}$.

Meanwhile, platinum markers include Visicoil MR manufactured by RadioMed Corporation/IBA (RadioMed Corporation; Bartlett, TN, USA/IBA; Schwarzenbruck, Germany) and LumiCoil manufactured by Boston Scientific (Marlborough, MA, USA). They are available in $0.35,0.46$, and $0.75 \mathrm{~mm}$ diameters and 5 and $10 \mathrm{~mm}$ lengths (Table 1). Platinum markers are considered to have better visibility on magnetic resonance imaging than gold markers because, theoretically, platinum has unpaired electrons in its outer shell; however, further investigations are needed to clinically prove this theory, and it should be considered that cone-beam CT is widely used in SBRT for pancreatic cancer when selecting fiducial markers according to material. ${ }^{27}$

Lastly, Acculoc Carbon Marker manufactured by Carbon Medical Technologies Inc. (Saint Paul, MN, USA) is available with a diameter of $1 \mathrm{~mm}$ and a length of $3 \mathrm{~mm}$ (Table 1). Carbon has a lower atomic number than gold or platinum. It has been shown to provide improved images in a study that used a high-resolution phantom model applied to one of the external beam radiotherapies (i.e., proton radiotherapy). However, the use of carbon markers in EUS-guided fiducial placement can be limited by their diameter, which can be as large as $1 \mathrm{~mm}$, and there have been only a few studies on the use of carbon markers in SBRT for pancreatic cancer. ${ }^{28}$

Slagowski et al. compared the visibility and artifacts of 11 commercially available fiducial markers in a virtual SBRT study using a phantom model. ${ }^{9}$ Of the fiducial markers, Visicoil MR ( $5 \mathrm{~mm}$ length, $0.75 \mathrm{~mm}$ diameter, coiled shape, platinum) showed the highest contrast, followed by Loose Gold Marker (3 mm length, $0.8 \mathrm{~mm}$ diameter, cylindrical shape, gold), EchoTip Ultra Fiducial Needle (5 mm length, $0.43 \mathrm{~mm}$ diameter, step shape, gold), and Gold Anchor (10 mm length, $0.28 \mathrm{~mm}$ diameter, ball shape, gold), in the order listed. ${ }^{9}$ In addition, fewer artifacts were produced by Acculoc Carbon Marker (3 mm length, $1 \mathrm{~mm}$ diameter, cylindrical shape, carbon), Gold Anchor (10 mm length, $0.28 \mathrm{~mm}$ diameter, linear shape, gold), and Visicoil MR (10 mm length, $0.35 \mathrm{~mm}$ diameter, coiled shape, platinum). ${ }^{9}$ Moreover, well-balanced images in terms of contrast and artifacts were observed with Beacon FNF Preloaded Needle (5 mm length, $0.43 \mathrm{~mm}$ diameter, cylindrical shape, gold) and Visicoil (10 mm length, 0.35 mm diameter, coiled shape, gold). ${ }^{9}$ However, the study results were derived from phantom models instead of live pancreatic tissues, and visibility was assessed without considering technical convenience or the incidence of complications. Larger diameters and longer lengths are associated with improved visibility but also with increased technical difficulty. A survey conducted among physicians showed that their most preferred fiducial markers were coil- or cylinder-shaped gold fiducials with diameters of $0.35-0.43 \mathrm{~mm}$ and lengths of 5-10 $\mathrm{mm}$ showing a balance between contrast and artifacts (Fig. 1).

\section{Liquid-type fiducial markers}

Despite their various advantages, fiducial markers made from metallic materials still have the following disadvantages: artifacts cannot be completely removed; there is a risk of migration; and the lesion tends to remain even after treatment if not surgically resected. Recently, there has been an attempt to create space between the tumor and adjacent tissues by injecting liquid gel, with the aim of reducing damage to adjacent tissues during radiation therapy for prostate cancer. ${ }^{29-32}$ The most frequently used liquid component is hydrogel (TraceIT Fiducial Marker; Augmenix Inc., Bedford, MA, USA) made of iodinated polyethylene glycol particles. ${ }^{29-32}$ Hydrogels, approved as soft-tissue fiducial markers by the FDA, remain in the body for 3 months, become absorbed intravitally at 7 months, and are finally cleared through the kidneys. ${ }^{33}$ Ussui et al. in 2016 demonstrated the feasibility of liquid hydrogels as fiducial markers in a study with two participants with pancreatic cancer. ${ }^{34}$ Theoretically, liquid fiducial markers do not produce artifacts typical with metal fiducials, have no injury risk to operators during fiducial loading, have a lower risk of migration, and disappear from the lesion by itself after treatment. However, there have been only a few studies using liquid fiducial markers, and other well-designed, comparative studies with other markers made of different materials are needed. ${ }^{35}$

\section{Shapes of fiducial markers}

Among the studies on fiducial markers, metal markers have been examined the most. Moreover, the studies similarly concluded that fiducials should have a shape that can aid the loading and placement of EUS-FNA needles cause less migration after insertion, and produce high contrast and fewer artifacts on imaging. Fiducial markers have been developed in several 


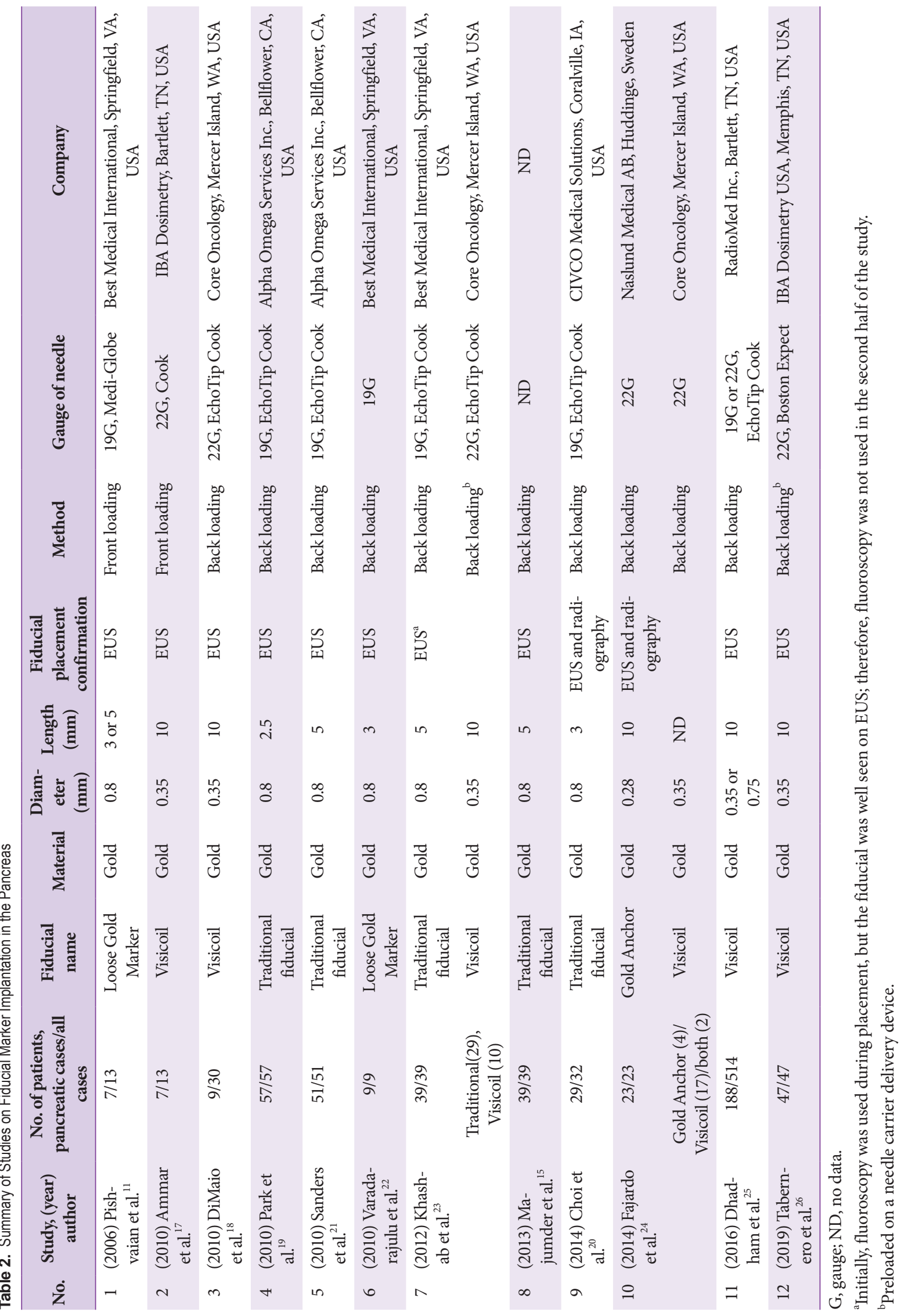




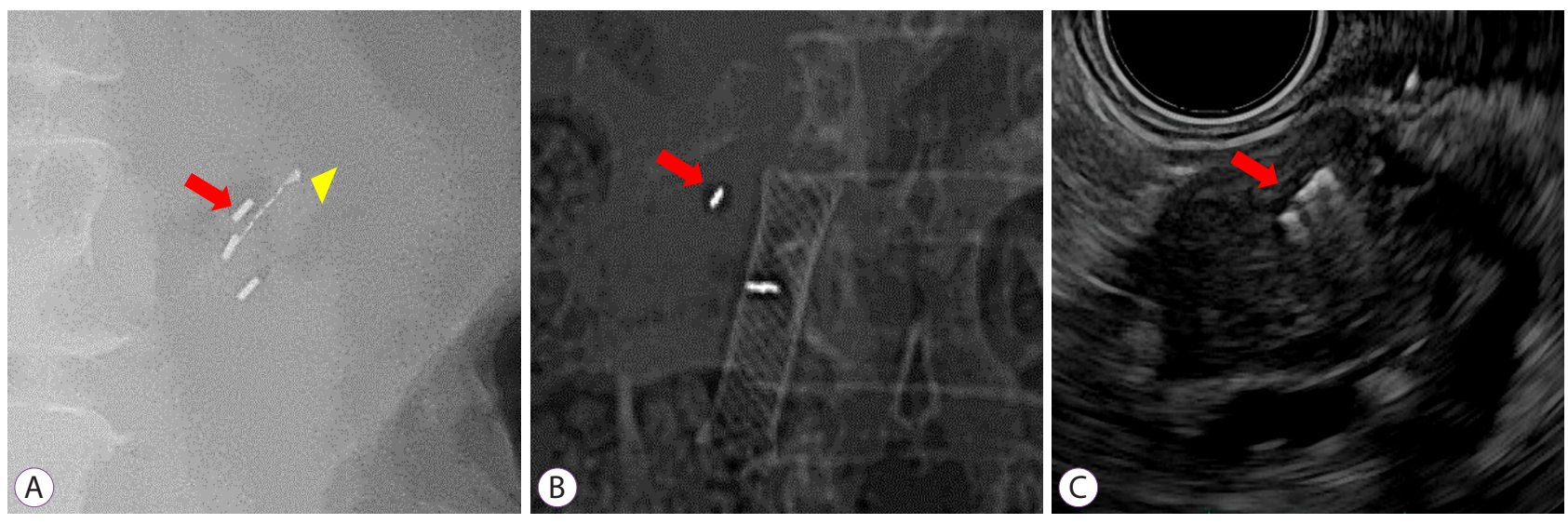

Fig. 1. Fluoroscopic and endosonographic views after EUS-guided fiducial placements. (A) Fluoroscopic view of Loose Gold Marker (0.80 mm diameter, $3 \mathrm{~mm}$ length; red arrow) and Gold Anchor (0.28 mm diameter, $20 \mathrm{~mm}$ length; yellow arrowhead). (B) Fluoroscopic view of Visicoil (0.35 mm diameter, $10 \mathrm{~mm}$ length; arrow). (C) Endosonographic view of Gold Anchor ( $0.28 \mathrm{~mm}$ diameter, $20 \mathrm{~mm}$ length; arrow).

shapes, most commonly cylindrical, linear, coiled, and step shapes, which may appear spherical in images depending on the level of transformation after insertion or the imaging angles (Table 1). Traditional fiducial markers were cylindrical in shape. Cylindrical markers are relatively easy to manufacture; however, they might have an increased risk of migration because their homogeneous shape tends to allow them to move easily. ${ }^{11,19,21}$ To allow fiducial markers to be inserted with a decreased migration risk, their shapes have been changed and the surface area in contact with the adjacent tissues has been increased.

Gold Anchor, a linear-shaped fiducial marker, was designed to have a lower risk of migration. When the FNA needle is withdrawn from the lesion, the multiple cutouts at regular intervals of Gold Anchor allow thinner parts of the marker to gradually fold and be imbedded with the adjacent tissues (Fig. $1 \mathrm{~A}, \mathrm{C}){ }^{2}$ As a linear-shaped fiducial marker with a surface area in the shape of a helical coil, Visicoil easily bends and changes its shape when inserted. Theoretically, this property allows Visicoil to be more firmly implanted in the lesion. Such high flexibility may enable the marker to adjust to the movements of adjacent tissues (Fig. 1B). In addition, Visicoil is known to have high stability and low migration risk because the helically coiled shape increases the surface area that comes into contact with soft tissue. ${ }^{17,18}$

The shapes of EchoTip Ultra Fiducial Needle and Beacon FNF Preloaded Needle are similar to those of the previous fiducial markers, but these FNA needles are preloaded with fiducial markers for operator convenience. The EchoTip Ultra Fiducial Needle is preloaded with four solid fiducial markers, which are step-shaped and resemble a cylinder with notches carved into them (Fig. 2A). ${ }^{13,36}$ The needle and fiducial design allows for the stylet to sequentially deploy the four fiducials, and the step-shaped structure may help reduce migration within tissues. The Beacon FNF Preloaded Needle is preloaded with two fiducial markers in a cylindrical configuration with knurls or ridges etched on their surface to decrease the risk of migration. ${ }^{2}$ Platinum Visicoil MR is shaped similar to a helical coil and resembles gold Visicoil in many respects, whereas LumiCoil has a coil-shaped surface and has two different configurations: straight and figure of eight (Fig. 2B).

\section{Techniques of EUS-guided fiducial placement}

The technique of EUS-guided fine-needle injection is the foundation of EUS-guided fiducial marker placement. ${ }^{13}$ The sizes of FNA needles used for EUS-guided fiducial placement are 19 and 22 gauge. The gauge of the FNA needle is determined by the diameter of the fiducial marker. For example, a fiducial marker with a diameter of $\geq 0.75 \mathrm{~mm}$ would need to be used with a 19-gauge needle. Traditional cylindrical markers that were used in the early years of EUS-guided fiducial placement had a diameter of $0.8 \mathrm{~mm}$ and were accordingly used with 19-gauge needles. However, inserting a 19-gauge FNA needle with high rigidity into a lesion located in the pancreatic head may be difficult to achieve using the transduodenal approach owing to the angulations of the EUS scope ${ }^{11,15,19-23}$ For this reason, 22-gauge FNA needles are preferred for use with fiducial markers with diameters $\leq 0.75 \mathrm{~mm}$ (i.e., 0.28 or $0.35 \mathrm{~mm}$ ). ${ }^{13,25}$ However, when using too small fiducials relative to the size of the FNA needle channel, the fiducials could fall out of the needle lumen or kinking could occur while deploying the fiducials with the stylet. Therefore, it is 

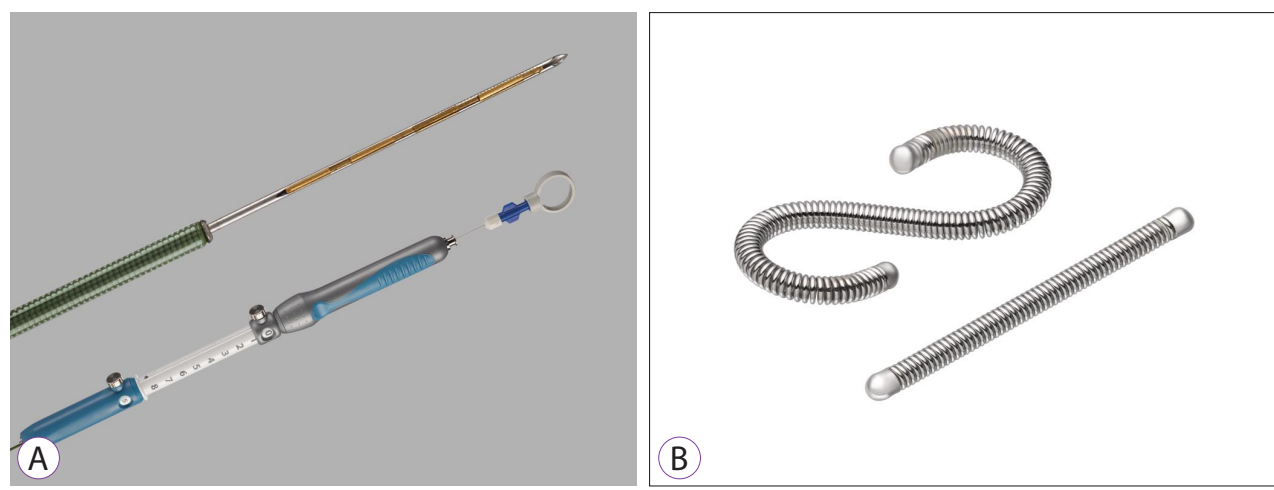

Fig. 2. Echo Tip Ultra Fiducial Needle and LumiCoil. (A) EchoTip Ultra Fiducial Needle is preloaded with four fiducial markers. (B) LumiCoil is a platinum fiducial marker with two different shapes: figure of eight and straight.
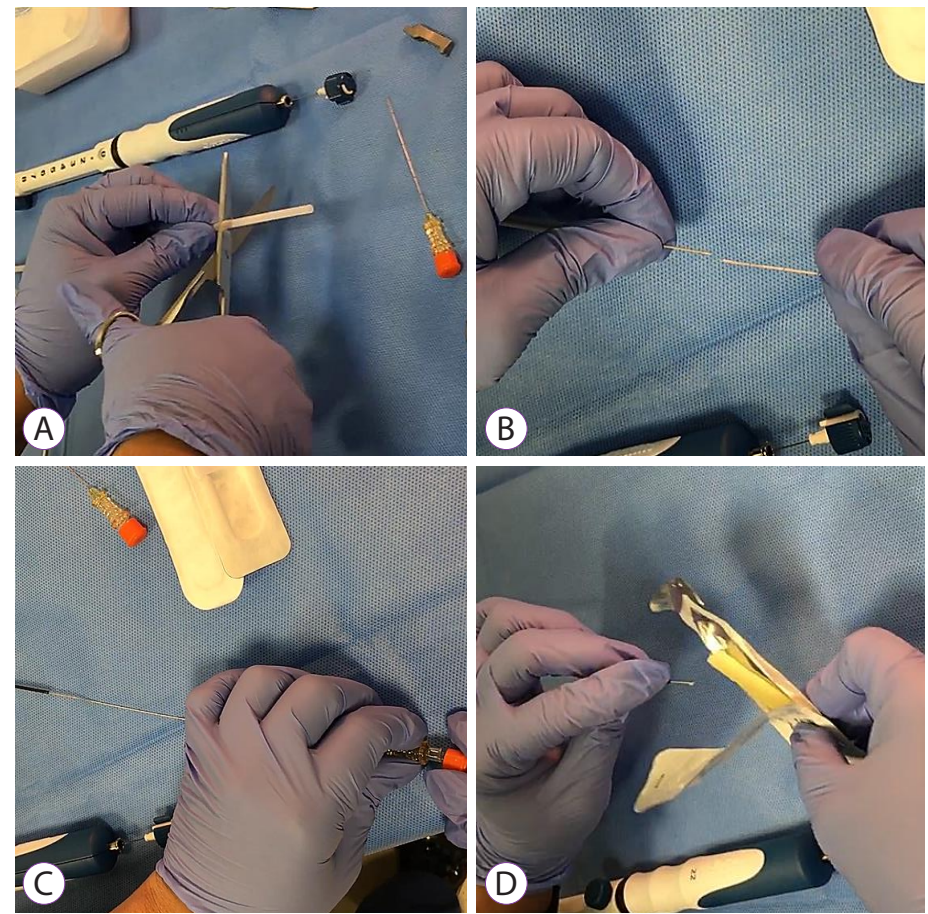

Fig. 3. Fiducial back-loading technique using a needle carrier delivery device. (A) Approximately $3 \mathrm{~cm}$ of the outer plastic sheath of the FNA needle package is cut with scissors. (B) The needle carrier delivery device is pushed into the FNA needle tip. (C) The fiducial loaded in the needle carrier delivery device is inserted into the FNA needle tip using a stylet. (D) The tip of FNA needle with the fiducial is sealed with bone wax. FNA, fine needle aspiration

advisable to use fiducials that can easily move within the FNA needle lumen and that fit the diameter of the FNA needle. ${ }^{24}$

There are two different approaches for loading a fiducial marker within the EUS-FNA needle: back-loading and front-loading techniques. In 2006, Pishvaian et al. performed a front-loading technique using the following steps: first, the
EUS-FNA needle was inserted into the lesion; second, the stylet was completely removed; third, a fiducial marker was inserted into the needle lumen; and finally, the fiducial marker was pushed with the stylet. ${ }^{11}$ Likewise, in 2010, Ammar et al. used the front-loading technique and suggested that the stylet should be left out as long as the length of the fiducial marker 
to prevent the fiducial marker from being inserted into nontarget lesions. ${ }^{17}$ However, the front-loading approach has disadvantages in that it can be challenging to deploy the fiducial marker with the stylet in cases of angulated FNA needles. In addition, air bubbles often enter the lesion through the FNA needle channel after stylet removal, which degrades the images and accordingly makes it difficult to confirm the success of the procedure.

Another approach for loading fiducial markers is the back-loading technique (Fig. 3). In the back-loading approach, before FNA needle insertion into the EUS scope channel, Owens et al. retracted the stylet by $10 \mathrm{~mm}$. Thereafter, they back-loaded the fiducial marker into the FNA needle using forceps and sealed the fiducial with sterile bone wax. ${ }^{37}$ The back-loading technique described above shortened the time to push the fiducial with a long FNA needle, which was considered a drawback of the front-loading approach, and reduced the possibility of introducing air into the lesion. However, the procedure for back-loading a fiducial into the FNA needle tip using forceps can be complicated, and there is a risk of needle stick injury to the operator. To overcome this drawback, DiMaio et al. in 2010 simplified the complicated procedure of loading fiducials into the FNA needle tip using forceps by preloading fiducial markers on the needle carrier delivery device. ${ }^{18}$ Unlike the front-loading approach, bone wax is used to seal the needle tip to avoid the loss of preloaded fiducial markers while inserting the FNA needle into the EUS scope in the back-loading technique. The disadvantages of using bone wax include the possibility of blocking the entry of the needle tip owing to its hardness and the theoretical potential of bone wax itself to remain in the lesion and cause granuloma formation. The "wet-fill technique" was introduced as a method to reduce the use of bone wax. With the wet-fill technique, the fiducial marker is back-loaded into the FNA needle filled with saline by placing the needle tip into sterile saline and pulling the stylet by approximately $10 \mathrm{~cm} .^{38}$

Both the front- and back-loading methods involve complicated and time-consuming procedures for loading the fiducials in the FNA needle. To solve this problem, EUS-FNA needles preloaded with two or four fiducials have been designed to enable multifiducial delivery. Two products of preloaded fiducial markers are currently available: EchoTip Ultra Fiducial Needle from Cook Medical and Beacon FNF Preloaded Needle from Medtronic. The EchoTip Ultra Fiducial Needle holds four fiducial markers loaded in a 22-gauge FNA needle, and each of the four markers is successively implanted depending on the depth of pushing by the stylet (Fig. 2A). ${ }^{36}$ Meanwhile, the Beacon FNF Preloaded Needle has a unique design in that two fiducial markers are preloaded in a 22-gauge FNA needle with a locking system at the tip of stylet, which prevents simultaneous deployment of the two markers. ${ }^{2}$ In a randomized controlled trial, Machicado et al. in 2019 compared the preloaded EchoTip Ultra Fiducial Needle with the back-loading technique using Visicoil. They found no significant differences in adverse events, migration rate, and visibility, but observed a decrease in procedure time with the EchoTip Ultra Fiducial Needle. $^{36}$

\section{Technical success rate of EUS-guided fiducial placement}

Chavalitdhamrong et al. in 2015 reviewed nine research articles and concluded that the technical success rate of EUS-guided fiducial placement was between $85 \%$ and $100 \% .{ }^{13}$ In 2020, Patel et al. analyzed 820 patients with pancreatic cancer selected from 11 studies and reported that the technical success rate of EUS-guided fiducial placement was $96.27 \%$ (95\% confidence interval [CI], 95.35-97.81). ${ }^{39}$ Although not limited to pancreatic cancer, Coronel et al. in 2019 analyzed 1155 patients with gastrointestinal malignancy selected from nine research articles and five abstracts. They estimated the technical success rate of EUS-guided fiducial placement to be 98\% (95\% CI, 96-99). ${ }^{16}$

\section{Technical difficulties of EUS-guided fiducial placement}

The technical difficulties of EUS-guided fiducial placement are broadly divided into three areas: operator characteristics, lesion attributes, and device characteristics. ${ }^{16,39}$ First, operator characteristics refer to the operator's proficiency level in implementing novel techniques. Park et al. found failure incidents in 12 of 57 cases in total and suggested that an adequate learning curve is required. ${ }^{19}$ The second area is the attributes of the lesion. In patients who have undergone surgeries such as pancreaticoduodenectomy, it can be difficult to locate the lesion using EUS. Moreover, if the lesion is located in the pancreatic head or uncinate process, a transduodenal approach would be required. In this case, using a 19-gauge FNA needle may make it more challenging to target the lesion because of the rigidity of the needle, and it may be more difficult to push fiducial markers through the FNA needle tip using the front-loading approach. In cases in which there are intervening vessels, the procedure is also challenging. Finally, device characteristics are divided into the characteristics of the EUS-FNA needle and those of the fiducial markers. As previously stated, the 22-gauge EUS-FNA needle is the recommended size, as it allows for more versatility in procedures. The characteristics of fiducial markers include their diameter, shape, and loading method. The optimal fiducial markers in terms of technical 
characteristics are those that are small in diameter, easily loaded into the FNA needle, and easily deployed by the stylet of the EUS-FNA needle into the lesion. The loading methods of EUS-FNA needles with fiducial markers can also contribute to technical difficulties. Recent studies have revealed that fiducials with diameters $\leq 0.75 \mathrm{~mm}$ that could be loaded into 22-gauge EUS-FNA needles are preferred; the back-loading technique is preferred over the front-loading technique; and preloading fiducial marker needles may reduce the procedure time and eliminate the risk of needle stick injury to operator. ${ }^{11,15,17-26}$

\section{Risk of migration of fiducial markers}

In 2020, Patel et al. performed a systematic review and meta-analysis of seven studies on EUS-guided fiducial marker placement for pancreatic cancer and reported a $4.33 \%$ migration rate of fiducial markers (95\% CI, 2.45-6.71). ${ }^{39}$ Similarly, Coronel et al., in a systematic review of five studies of EUS-guided fiducial placement for gastrointestinal malignancy, reported a migration rate of fiducial markers of 3\% (95\% CI, 1.0-8.0). ${ }^{16}$ Depending on the onset time, fiducial migration is divided into immediate migration and delayed migration. ${ }^{21}$ Immediate fiducial migration may occur owing to technical difficulties, and an additional fiducial marker can be inserted once the migration is detected by the operator. Delayed fiducial migration can have three main causes: 1) changes in tissue related to tumor regression resulting from neoadjuvant chemotherapy; 2) relocation of fiducial markers induced by bleeding, swelling, or inflammation in the tissue due to fiducial insertion into the lesion; and 3) infiltration of air bubbles into the lesion along with the fiducial marker, leading to delayed detection of the precise location of the marker after its EUS-guided insertion. ${ }^{20,39}$ In the strict sense, the third case is seldom distinguished from incorrect placement occurring at the point of EUS-guided fiducial placement. To avoid the third case, caution is required to avoid the inclusion of an air bubble within the EUS-FNA needle while loading fiducial markers. ${ }^{20,39}$ For accurate targeting, efforts should be made to reduce fiducial migration during SBRT, although no adverse events linked with migration have been reported in the existing studies. ${ }^{16}$ Factors assumed to contribute to fiducial migration may include the shape of the fiducial markers. Kerdsirichairat et al. reported that the fiducial markers of Beacon FNF Preloaded Needle have a higher risk of migration than Visicoil and Gold Anchor fiducial markers, with Gold Anchor having the lowest risk. ${ }^{2,40}$ This can be interpreted to mean that the cylindrical type, which has the smallest surface area in contact with the adjacent tissues, would have the highest risk of migration; therefore, Gold Anchor, which folds after insertion, would have the lowest risk of migration. Furthermore, the fiducial marker of Beacon FNF Preloaded Needle $(5 \mathrm{~mm}$ length) is shorter than the other fiducial markers included in the study, which may have resulted in a higher migration rate.

\section{Adverse events of EUS-guided fiducial placement}

A systematic review of 10 studies that included only patients with pancreatic cancer reported that the complication rate of EUS-guided fiducial implantation was 4.85\% (95\% CI, 3.04-7.03)..$^{39}$ Although the systematic review conducted by Coronel et al. included patients with all types of gastrointestinal malignancies, their meta-analysis produced similar results and showed a $4 \%$ adverse rate of EUS-guided fiducial implantation. ${ }^{16}$ However, lethal or life-threatening bleeding cases have not been reported. The complications reported in the study mostly included minor bleeding, mild pancreatitis, and infection. Abdominal pain, vomiting, and needle malfunction have also been reported; however, no study evaluating long-term complications has been published. ${ }^{12,13}$

Since the publication of an early report on cholangitis after EUS-guided fiducial placement for pancreatic cancer, prophylactic antibiotics have been widely used and no further cases of lethal infectious complications have been reported. ${ }^{15,16,18-21,23}$

\section{CONCLUSIONS}

From the outcomes of the existing studies, it is apparent that EUS-guided fiducial marker placement plays a beneficial role in SBRT for pancreatic cancer. Well-designed studies comparing techniques of EUS-guided fiducial placement or comparing fiducial markers have not been conducted. Future comparative studies are needed to determine the optimal types of metallic material or the shape of fiducials, as well as the optimal delivery technique. Additionally, a newly introduced liquid hydrogel has started to receive attention in terms of whether it would be able to replace metallic materials in the development of fiducial markers. However, the most important factor is close cooperation and discussion about the selection of fiducial markers for each patient among gastroenterologists, oncologists, surgeons, and radiation oncologists who perform SBRT.

Conflicts of Interest

The authors have no potential conflicts of interest.

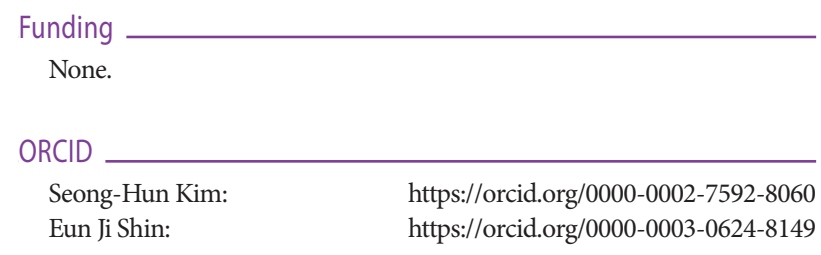




\section{REFERENCES}

1. Siegel RL, Miller KD, Jemal A. Cancer statistics, 2020. CA Cancer J Clin 2020;70:7-30.

2. Kerdsirichairat T, Shin EJ. Role of endoscopic ultrasonography guided fiducial marker placement in gastrointestinal cancer. Curr Opin Gastroenterol 2020;36:402-408.

3. Gurka MK, Kim C, He AR, et al. Stereotactic body radiation therapy (SBRT) combined with chemotherapy for unresected pancreatic adenocarcinoma. Am J Clin Oncol 2017;40:152-157.

4. Conroy T, Bachet J-B, Ayav A, et al. Current standards and new innovative approaches for treatment of pancreatic cancer. Eur J Cancer 2016;57:10-22.

5. Chauffert B, Mornex F, Bonnetain F, et al. Phase III trial comparing intensive induction chemoradiotherapy (60 Gy, infusional 5-FU and intermittent cisplatin) followed by maintenance gemcitabine with gemcitabine alone for locally advanced unresectable pancreatic cancer. Definitive results of the 2000-01 FFCD/SFRO study. Ann Oncol 2008;19:15921599.

6. Loehrer PJ, Feng Y, Cardenes H, et al. Gemcitabine alone versus gemcitabine plus radiotherapy in patients with locally advanced pancreatic cancer: an eastern cooperative oncology group trial. J Clin Oncol 2011;29:4105-4112.

7. de Geus SWL, Eskander MF, Kasumova GG, et al. Stereotactic body radiotherapy for unresected pancreatic cancer: a nationwide review. Cancer 2017;123:4158-4167.

8. Petrelli F, Comito T, Ghidini A, Torri V, Scorsetti M, Barni S. Stereotactic body radiation therapy for locally advanced pancreatic cancer: a systematic review and pooled analysis of 19 trials. Int J Radiat Oncol Biol Phys 2017;97:313-322.

9. Slagowski JM, Colbert LE, Cazacu IM, et al. Evaluation of the visibility and artifacts of 11 common fiducial markers for image guided stereotactic body radiation therapy in the abdomen. Pract Radiat Oncol 2020;10:434-442.

10. Moningi S, Abi Jaoude J, Kouzy R, et al. Impact of fiducial marker placement before stereotactic body radiation therapy on clinical outcomes in patients with pancreatic cancer. Adv Radiat Oncol 2021;6:100621.

11. Pishvaian AC, Collins B, Gagnon G, Ahlawat S, Haddad NG. EUS-guided fiducial placement for CyberKnife radiotherapy of mediastinal and abdominal malignancies. Gastrointest Endosc 2006;64:412-417.

12. Bhutani MS, Herman JM. Endoscopic ultrasound-guided fiducial placement for gastrointestinal malignancies. Gastroenterol Hepatol (N Y) 2019;15:167-170.

13. Chavalitdhamrong D, DiMaio CJ, Siersema PD, Wagh MS. Technical advances in endoscopic ultrasound-guided fiducial placement for the treatment of pancreatic cancer. Endosc Int Open 2015;3:E373-E377.

14. Han J, Chang KJ. Endoscopic ultrasound-guided direct intervention for solid pancreatic tumors. Clin Endosc 2017;50:126-137.

15. Majumder S, Berzin TM, Mahadevan A, et al. Endoscopic ultrasound-guided pancreatic fiducial placement: how important is ideal fiducial geometry? Pancreas 2013;42:692-695.

16. Coronel E, Cazacu IM, Sakuraba A, et al. EUS-guided fiducial placement for GI malignancies: a systematic review and meta-analysis. Gastrointest Endosc 2019;89:659-670.e18.

17. Ammar T, Coté GA, Creach KM, Kohlmeier C, Parikh PJ, Azar RR. Fiducial placement for stereotactic radiation by using EUS: feasibility when using a marker compatible with a standard 22-gauge needle. Gastrointest Endosc 2010;71:630-633.

18. DiMaio CJ, Nagula S, Goodman KA, et al. EUS-guided fiducial placement for image-guided radiation therapy in GI malignancies by using a 22-gauge needle (with videos). Gastrointest Endosc 2010;71:1204-1210.

19. Park WG, Yan BM, Schellenberg D, et al. EUS-guided gold fiducial insertion for image-guided radiation therapy of pancreatic cancer: 50 successful cases without fluoroscopy. Gastrointest Endosc 2010;71:513-
518

20. Choi J-H, Seo D-W, Park DH, Lee SK, Kim M-H. Fiducial placement for stereotactic body radiation therapy under only endoscopic ultrasonography guidance in pancreatic and hepatic malignancy: practical feasibility and safety. Gut Liver 2014;8:88-93.

21. Sanders MK, Moser AJ, Khalid A, et al. EUS-guided fiducial placement for stereotactic body radiotherapy in locally advanced and recurrent pancreatic cancer. Gastrointest Endosc 2010;71:1178-1184.

22. Varadarajulu S, Trevino JM, Shen S, Jacob R. The use of endoscopic ultrasound-guided gold markers in image-guided radiation therapy of pancreatic cancers: a case series. Endoscopy 2010;42:423-425.

23. Khashab MA, Kim KJ, Tryggestad EJ, et al. Comparative analysis of traditional and coiled fiducials implanted during EUS for pancreatic cancer patients receiving stereotactic body radiation therapy. Gastrointest Endosc 2012;76:962-971.

24. Dávila Fajardo R, Lekkerkerker SJ, van der Horst A, et al. EUS-guided fiducial markers placement with a 22-gauge needle for image-guided radiation therapy in pancreatic cancer. Gastrointest Endosc 2014;79:851855

25. Dhadham GC, Hoffe S, Harris CL, Klapman JB. Endoscopic ultrasound-guided fiducial marker placement for image-guided radiation therapy without fluoroscopy: safety and technical feasibility. Endosc Int Open 2016;4:E378-E382.

26. Tabernero S, Prados S, Rubio MDC, de la Morena F, López M, Sánchez E. Endoscopic ultrasound-guided fiducial placement in pancreatic tumors: safety and technical feasibility. Rev Esp Enferm Dig 2019;111:425-430.

27. Nair VJ, Szanto J, Vandervoort E, et al. Feasibility, detectability and clinical experience with platinum fiducial seeds for MRI/CT fusion and real-time tumor tracking during CyberKnife ${ }^{\star}$ stereotactic ablative radiotherapy. J Radiosurg SBRT 2015;3:315-323.

28. Gleeson FC, Tryggestad EJ, Remmes NB, et al. Knowledge of endoscopic ultrasound-delivered fiducial composition and dimension necessary when planning proton beam radiotherapy. Endosc Int Open 2018;6:E766-E768

29. Hamstra DA, Mariados N, Sylvester J, et al. Continued benefit to rectal separation for prostate radiation therapy: final results of a phase III trial. Int J Radiat Oncol Biol Phys 2017;97:976-985.

30. Rao AD, Feng Z, Shin EJ, et al. A novel absorbable radiopaque hydrogel spacer to separate the head of the pancreas and duodenum in radiation therapy for pancreatic cancer. Int J Radiat Oncol Biol Phys 2017;99:11111120.

31. Rao AD, Shin EJ, Beck SE, et al. Demonstration of safety and feasibility of hydrogel marking of the pancreas-duodenum interface for image guided radiation therapy (IGRT) in a porcine model: implications in igrt for pancreatic cancer patients. Int J Radiat Oncol Biol Phys 2018;101:640-645.

32. Kerdsirichairat T, Narang AK, Thompson E, et al. Feasibility of using hydrogel spacers for borderline-resectable and locally advanced pancreatic tumors. Gastroenterology 2019;157:933-935.

33. de Souza Lawrence L, Ford E, Gilbert C, et al. Novel applications of an injectable radiopaque hydrogel tissue marker for management of thoracic malignancies. Chest 2013;143:1635-1641.

34. Ussui V, Kuritzky N, Berzosa M. Mo1992 EUS-guided liquid fiducial placement for stereotactic radiotherapy in pancreatic cancer: feasibility study. Gastrointestinal Endoscopy 2016;83:AB486-AB487.

35. Ussui V, Kuritzky N, Berzosa M. EUS-guided liquid fiducial placement for stereotactic radiotherapy in pancreatic cancer: feasibility study. Endosc Ultrasound 2018;7:135-136.

36. Machicado JD, Obuch JC, Goodman KA, et al. Endoscopic ultrasound placement of preloaded fiducial markers shortens procedure time compared to back-loaded markers. Clin Gastroenterol Hepatol 2019; 17:2749-2758.e2.

37. Owens DJ, Savides TJ. EUS placement of metal fiducials by using a backloaded technique with bone wax seal. Gastrointest Endosc 2009;69:972- 
973.

38. Khara HS, Pineda-Bonilla JJ, Chaput KJ, Johal AS. Endoscopic ultrasound-guided placement of fiducial markers using a novel "wet-fill technique" without a bone wax seal. Endoscopy 2013;45 Suppl 2 UCT$\mathrm{N}: \mathrm{E} 426-\mathrm{E} 427$.

39. Patel JB, Revanur V, Forcione DG, Bechtold ML, Puli SR. Endoscopic ultrasound-guided fiducial marker placement in pancreatic cancer: a systematic review and meta-analysis. World J Gastrointest Endosc 2020;12:231-240.

40. Kerdsirichairat T, Kim S-H, Narang A, et al. Mo1276 Visibility, artifact and migrations using three types of fiducials for pancreatic ductal adenocarcinoma (PDAC) patients receiving stereotactic body radiation therapy (SBRT). Gastrointestinal Endoscopy 2018;87:AB426. 